difference was also noted with the onset of seizure parameter among group I (control for PTZ) in comparison with groups III and IV $(p<0.001)$. All the above parameters were comparable to the standard group II in respect to the groups III and IV with p-value more than 0.05. In MES model, the parameters like (scores and THLE [tonic hind limb extension]) were also statistically significant different among groups VII and VIII when compared with the control group (group V) for MES with p-value less than 0.05 . The above two parameters were also comparable to the standard drug group (group VI) for MES model in respect to the experimental test drug groups (group VII and VIII) with p-value more than 0.05.

Conclusion: $\mathrm{Na}+-\mathrm{K}^{+}-2 \mathrm{Cl}^{-}$cotransport inhibitors (furosemide and torasemide) exhibited significant antiseizure activities in chemically ( $p \mathrm{TZ}$ ) and electrically (MES) induced seizure model tests in Wistar albino rats.

\section{A009: Correlation between Distance from Health Centre and Adherence to Clinic Appointments and Medication Procurement among People with Epilepsy in the Community \\ Chirag Gupta, ${ }^{1}$ Gagandeep Singh, ${ }^{1}$ Karan Chouhan, ${ }^{1}$ Archita Chawla, ${ }^{1}$ R.K. Setia, ${ }^{1}$ Suman Sharma, ${ }^{1}$ Namita Bansal ${ }^{1}$ 'Dayanand Medical College and Hospital, Ludhiana, Punjab, India}

Introduction: In resource limited countries (e.g., India), distance from the health care facility may be a barrier to treatment adherence in people with epilepsy.

Objective: To study the relationship between Euclidean distance of home dwelling from the health care center and number of health center visits to procure medication and medical advice by people with epilepsy.

Methods: The setting of the study was a community-based project in which subjects from across the Ludhiana city in one arm visited the civil hospital on a monthly basis for consultation and to procure antiepileptic drugs. We mapped out the homes of people with epilepsy who attended the epilepsy clinic and calculated the Euclidean distance between each home and the civil hospital. We studied the association between clinic visits and the distance factor; age, gender, educational and economic status, and duration of epilepsy as covariates.

Results: The mean distance between home and hospital was $3.33 \pm 0.18 \mathrm{~km}$. The average calculated cost of antiepileptic drugs per month was Rs.303 only. We could not establish a direct correlation between the distance and adherence $(p$-value $=0.74)$, although socioeconomic status $(p=0.175)$ and gender $(p=0.113)$ were associated with adherence.

Conclusion: The study is ongoing and although at this stage a correlation between distance and clinic visits could not be established, longer follow-up may demonstrate the distance decay effect.
A0010: Clinicoradiological Evaluation of Newly Diagnosed Epilepsy: A Monocentric Prospective Study from a Tertiary Care Hospital of Eastern India

Panigrahy D., ${ }^{1}$ Mallick A.K., ${ }^{1}$ Mohanty G., ${ }^{1}$ Samant M. ${ }^{1}$

${ }^{1}$ SCB Medical College and Hospital, Cuttack, Odisha, India

Introduction: "Newly diagnosed epilepsy" is defined as a subset of epileptic disorder, which presented or diagnosed first time during study period, and had two or more than two episodes of seizure. Early evaluation and diagnosis of Epilepsy is very important for better treatment.

Aim: Aim of study was to evaluate epidemiological, clinical profile, as well as radiologic characteristics in cases of newly diagnosed epilepsy and to find out correlation between them.

Materials and Methods: It was a prospective and descriptive study of 1-year duration, conducted in the department of neurology in Sriram Chandra Bhanja Medical College and Hospital, Cuttack, Odisha, India. Three hundred newly diagnosed epilepsy patients, more than 5 years of age, were included and subjected to detail clinical and radiological evaluation.

Results: Majority patients in our study were from second and third decades (mean age $=25$ years, $S D=11.04$ ) with characteristic male preponderance. Most patients were from rural Background: and majority of them (60\%) attended neurology clinic after 3 to 10 episodes of seizures. Seizures in awake state and seizures without precipitating factors were more common. Headache was the most common prodromal symptom. Generalized seizure dominated over focal seizure (62:34) with tonic-clonic type (66.67\%) being most common in the generalized seizure cohort. Focal seizure with secondary generalization was seen in $70.5 \%$ cases. Drowsiness (38\%) and Todd's palsy (6\%) were most and least common postictal symptoms in our study. Our study also revealed abnormal computed tomography (CT) findings in $70.6 \%$ of focal seizures and $24.2 \%$ of generalized seizures. Magnetic resonance imaging (MRI) was abnormal in 53.6\% of cases. Granulomatous lesion was dominant neuroimaging finding in our study.

Conclusion: This study concluded that males of second and third decades from rural Background are highly prone to epilepsy. Infectious causes like neurocysticercosis and tuberculoma are predominant etiologies in our area. Neuroimaging plays an important role in establishing and localizing etiology of seizure. Any patient coming with history of epilepsy or suspected as a case of newly diagnosed epilepsy must be investigated with neuroimaging for further management.

A0011: Changing Trends of Subacute Sclerosing Panencephalitis- Pre- and Postvaccination Era, Why Should Children Vaccinated against Measles Develop SSPE? Ekta Agarwal,' ${ }^{1}$ Srivastava K., ${ }^{1}$ Rajadhyaksha S. ${ }^{1}$

${ }^{1}$ Bharati Vidyapeeth Medical College Hospital and Research Centre, Pune, Maharashtra, India

Introduction: Subacute sclerosing panencephalitis (SSPE) is thought to occur several years after measles infection. Measles vaccine is protective against SSPE. However, 
SSPE has been reported in vaccinated children without prior history of measles infection.

Materials and Methods: A retrospective study was done to analyze vaccinated children with SSPE without prior history of measles infection.

Results: Fifteen out of 22 cases of SSPE in the past 10 years were immunized for measles. Mean age of onset was 8.4 years with $80 \%$ of children being under 10 years of age. Average time of progression to advanced stage of the disease was 2.5 months -3 months (range: 15 days -5 months).

Discussion In vaccinated children, a subclinical or unrecognized measles infection is speculated to be the source of wild virus, with exposure occurring before vaccination. Earlier the age of exposure, higher is the risk of SSPE. Although thought to be slowly progressive, recent trend indicates a younger age of onset and faster progression of SSPE from onset to critically disabling stages. Vaccination with measles at 9 months changes the epidemiology of SSPE leaving younger infants susceptible to measles. Also, as per previous studies, maternally acquired antibodies probably wane off by 3 to 4 months. With current immunization schedule of administering first dose of measles vaccine at 9 months, children less than 9 months remain susceptible to measles and later SSPE. However, vaccination before 9 months is found to be both effective and safe.

Conclusion: This study proposes a relook at the need of reducing the age of vaccination against measles to 6 months.

\section{A0012: Network Changes in Eating Epilepsy: An MEG Study} Bhargava G.K., ${ }^{1,2}$ Mariyappa N., ${ }^{1}$ Shishir D., ${ }^{2}$ Raghavendra K., ${ }^{2}$ Ravindranadh C.M., ${ }^{2}$ Bharath R.D., ${ }^{3}$ Saini J.S., ${ }^{3}$

Sanjib Sinha ${ }^{1,2}$

${ }^{1}$ MEG Research Lab, National Institute of Mental Health and Neurosciences, Bengaluru, Karnataka, India

2Department of Neurology, National Institute of Mental Health and Neurosciences, Bengaluru, Karnataka, India

${ }^{3}$ Department of Neuroimaging and Interventional Radiology, National Institute of Mental Health and Neurosciences, Bengaluru, Karnataka, India

Objective: To examine the network differences in eating epilepsy at resting state and during eating period using MEG recording.

Methodology Resting state MEG data were analyzed in 17 patients with eating epilepsy $(\mathrm{M}: \mathrm{F}=12: 5$, age at onset $=9.66 \pm 8.63$ years, age at evaluation $=20.6 \pm 8.7$ years $)$. All underwent presurgical workup including MRI, EEG, video-EEG, MEG, and neuropsychological assessment. MEG recording while eating (rice) was obtained in five patients $(\mathrm{M}: \mathrm{F}=2: 3)$. MRI (brain) showed normal-9; left perisylvian gliosis-4, bilateral peri-sylvian gliosis-2, left MTS-1, and left temporal astrocytoma-1. Data were down sampled to $500 \mathrm{~Hz}$, artifacts removed and bandpass filtered between 0.5 and 150 $\mathrm{Hz}$. Data were transformed to source space by beam forming and parcellated. Amplitude envelope correlation was applied to six frequency bands (delta: $1-4 \mathrm{~Hz}$, theta: 5-7 Hz, $\sigma: 8-12$ $\mathrm{Hz}, \beta$ : 13-30 Hz, gamma1: 30-60 Hz, gamma 2: 60-90 Hz) to derive adjacency matrices for both conditions. Network measures (clustering coefficient, modularity, local efficiency, betweenness centrality, degree, and path length) were derived using graph theory. Group comparisons were FDR corrected.

Results: Resting state networks showed increased clustering and pathological local information processing in the superior, inferior, and medial parietal regions and prefrontal regions predominantly in the $\beta$ and gamma frequency bands $(p<0.036)$. While eating, network changes involved the precuneus, inferior parietal, right prefrontal, bilateral calcarine, and entorhinal regions in the $\beta$ and gamma bands $(p<0.027)$.

Conclusion: Previous electrophysiological and functional imaging studies have implicated involvement of perisylvian region. Pathological information processing was noted across the perisylvian regions dominantly on the right hemisphere in this study. Eating resulted in recruitment of bilateral hubs between the parietal, temporal, and occipital structures.

\section{A0013: Impact of Epileptic Seizures in the Neurological Intensive Care Unit (NICU) on Glasgow Coma Scale (GCS) Singh G., ${ }^{1}$ Paul B.S., ${ }^{1}$ Chaudhary A.K., ${ }^{1}$ Singh G. ${ }^{1}$ \\ 'Dayanand Medical College and Hospital, Ludhiana, Punjab, India}

Aims: To determine the frequency, magnitude, range, and duration of change in GCS in the aftermath of seizures occurring in the neurological intensive care unit.

Methods: Subjects were enrolled if they had a seizure during admission to a neurological intensive care unit. Their last recorded GCS prior to the seizures was extracted from chart review and GCS monitored prospectively at 2, 4, 8, 24, and 48 hours after the seizure. For comparison, subjects with matching GCS scores were also monitored for 48 hours to document fluctuations in their scores.

Results: Mean preseizure GCS in cases $(n=50)$ was $(10 \pm 4)$ and was similar to controls $(n=50)(10 \pm 4)$. Immediately after the seizure, the mean GCS fell to (6 \pm 3$)$ and thereafter increased progressively at 2 hours $(8 \pm 4), 4$ hours ( $9 \pm$ 4), 8 hours ( $9 \pm 4$.), 12 hours ( $9 \pm 5$ ), 24 hours ( $9 \pm 5$ ), and 48 hours $(9 \pm 5)$. However, the mean GCS did not return to preseizure values even after 48 hours. No deviations were noted in the control group. At the end of 2 hours, 37 cases still had deranged GCS; end of 4 hours, 26 patients had deranged GCS; at 8 hours, 18 patients had deranged GCS; at 12 hours and 24 hours, 16 patients; and finally, at 48 hours, 13 patients still had deranged GCS.

Conclusion: Seizures in the ICU lead to drop in GCS of those affected and this drop may persist even up to 48 hours. This may confound the assessment of GCS. 